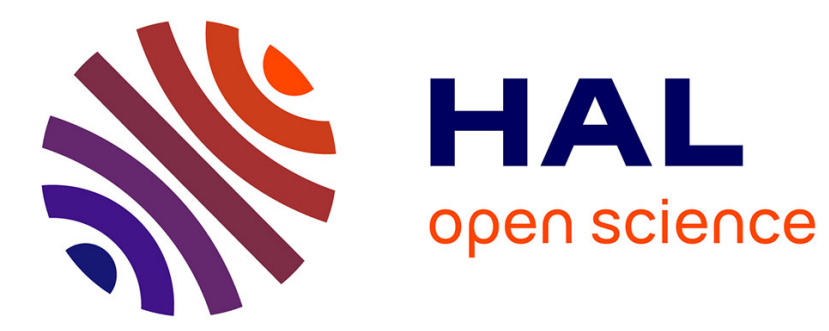

\title{
Les ordinaires liturgiques comme sources pour l'historien du Moyen Âge. À propos d'ouvrages récents
}

Eric Palazzo

\section{To cite this version:}

Eric Palazzo. Les ordinaires liturgiques comme sources pour l'historien du Moyen Âge. À propos d'ouvrages récents. Revue Mabillon, revue internationale d'histoire et de littérature religieuses, 1992, 3, pp.233-240. 10.1484/J.RM.2.305514 . halshs-01355091

\section{HAL Id: halshs-01355091 https://shs.hal.science/halshs-01355091}

Submitted on 22 Aug 2016

HAL is a multi-disciplinary open access archive for the deposit and dissemination of scientific research documents, whether they are published or not. The documents may come from teaching and research institutions in France or abroad, or from public or private research centers.
L'archive ouverte pluridisciplinaire HAL, est destinée au dépôt et à la diffusion de documents scientifiques de niveau recherche, publiés ou non, émanant des établissements d'enseignement et de recherche français ou étrangers, des laboratoires publics ou privés. 
ecclesiae?" That had been the question of the Catholic bishop of Stockholm, Dr Brandenburg, at the beginning of the symposium. There is not yet a clear and definite answer, but the symposium certainly has given a contribution, preparing the ground for an answer.

\section{Alf HäRDELIN}

\section{LES ORDINAIRES LITURGIQUES COMME SOURCES POUR L'HISTORIEN DU MOYEN AGE. A PROPOS D'OUVRAGES RÉCENTS ${ }^{1}$}

Le fascicule 56 de la célèbre Typologie des sources du Moyen Age occidental, paru en 1991, est consacré à trois types de livres liturgiques : les ordines, les ordinaires et les cérémoniaux ${ }^{2}$. Après Michel Huglo pour les livres de chant (fasc. 52) et Joseph Szövérffy pour les hymnes (fasc. 55) - auxquels l'on peut ajouter dom Jacques Dubois pour les martyrologes (fasc. 26) -, Mgr Martimort, grand liturgiste français de ce siècle, offre aux médiévistes de tout bord un bel ouvrage de synthèse portant sur trois catégories de sources liturgiques. A l'heure où l'on reparle beaucoup d'histoire de la liturgie, de sa typologie documentaire, de sa place au sein des sciences historiques, l'ouvrage.de Mgr Martimort offre une bonne opportunité pour réfléchir sur les orientations prises par les historiens de la liturgie, notamment les spécialistes des livres.

J'aborderai trois points principaux : tout d'abord, les questions de typologie générale, notamment de définition du livre liturgique par rapport à d'autres sources du Moyen Age, que pose le choix de regrouper dans un même fascicule trois types de livres; en complément de l'ouvrage de A.-G. Martimort et après examen d'un certain nombre d'ordinaires médiévaux, j'essayerai ensuite de mieux comprendre l'histoire de ce type de livre, son origine, les raisons de son apparition, en particulier à la lumière de l'histoire des coutumiers monastiques, et d'affiner la typologie en vue de l'établissement d'une typologie de la célébration; enfin, j'examinerai le rôle des ordinaires dans l'histoire de l'Église et de la liturgie, son intérêt pour l'historien, l'historien de l'art, l'archéologue.

\section{Les ordinaires et la typologie des livres liturgiques}

A première vue, le néophyte ou le spécialiste pourront trouver normal de voir regroupés dans un même volume des documents - appelons-les ainsi pour le moment - liturgiques, tous trois de nature descriptive. Les ordines (romani) sont en effet des textes où sont décrits, voire prescrits, certains actes liturgiques, notamment les rituels, combinant rubriques et textes euchologiques (textes sacrés) qui servaient dans le déroulement même du culte. On sait qu'au cours du haut Moyen Age ils ont circulé sous forme de collections, ou bien étaient insérés dans d'autres livres liturgiques (comme le sacramentaire), puis ont été regroupés et sélectionnés pour former un livre liturgique à part entière : le pontifical, livre de l'évêque. Je ne m'étendrai pas sur les cérémoniaux qui constituent une sorte

1. Ce texte est, à peu de chose près, celui de la conférence prononcée lors des " Rencontres entre historiens et liturgistes " organisées à Paris par l'Institut supérieur de Liturgie, le 21 janvier 1992. Je remercie les intervenants du débat pour leurs remarques et suggestions dont j'ai essayé de tenir compte dans cette chronique.

2. A.-G. Martimort, Les " ordines \#, les ordinaires et les cérémoniaux, Turnhout, 1991 (Typologie des sources du Moyen Age occidental, fasc. 56). 
d'aboutissement de l'évolution typologique des ordinaires à une époque (la fin du Moyen Age, le Xv et le Xvi siècle, et même après) où les livres du culte sont entrés dans une autre phase de leur histoire, sans lien étroit avec celle qui a vu naître les ordinaires. L'ordinaire liturgique appartient, lui, à la catégorie de ce que j'appellerai les usuels de la liturgie, au même titre que les coutumiers, les recueils de règles, ou même ces petits "guides de lectures " (pour la lecture au réfectoire) que Donatella Nebbiai-Dalla Guarda a mieux fait connaître ${ }^{3}$.

Ces documents, dont les ordinaires, répondent au souci de codification des usages réguliers ou séculiers et apparaissent donc comme des compléments indispensables aux livres liturgiques stricto sensu. Je sais bien toutes les réserves, par ailleurs justifiées, que l'on doit avoir face à la définition de Victor Leroquais - reprise par Cyrille Vogel et bien d'autres - d'un livre liturgique : c'est le livre qui sert dans l'exercice du culte. Cette définition, restrictive et dangereuse si on la systématise, a surtout l'inconvénient majeur d'exclure bon nombre de documents (comme les ordinaires) qui ne se comprennent qu'à partir de la liturgie et de ses livres.

Dans son ouvrage, A.-G. Martimort établit une nette distinction entre coutumier et ordinaire (p. 66-67) :

"En pratique ce qui différencie aussitôt l'un et l'autre type de livre, c'est que l'ordinaire décrit le cours de l'année liturgique avec ses célébrations : office, messe, processions, tandis que le coutumier détaille [j'ajouterai : aussi] les usages et les rites de la vie de la communauté, précisant et complétant, s'il s'agit de moines ou de chanoines réguliers, les prescriptions de la Règle (...)."

Il existe néanmoins un lien réel et même presque génétique entre ces deux types de documents. On peut dire, en quelque sorte, que l'ordinaire est déjà contenu dans le coutumier (à propos du Liber tramitis de Cluny du xi ${ }^{e}$ siècle, A.-G. Martimort parle, à juste titre je crois, de premier ordinaire monastique), si bien qu'il eût été préférable, à mon sens, dans le cadre d'ụ fascicule de la Typologie de Louvain, de traiter des coutumiers à la place des ordines, dont on peut se demander si le lien typologique avec l'ordinaire est aussi profond. Dans les deux cas (ordinaires, ordines), il s'agit de livres descriptifs, voire prescriptifs, mais les ordines trouvent leur prolongement logique dans le pontifical, livre liturgique à part entière, et dans une bien moindre mesure dans le missel. Il semble donc qu'on aurait pu traiter des ordines romani et du pontifical dans un même volume.

Ces raisons propres à la matière liturgique et typologique ne sont souvent pas celles qui déterminent le plan d'une collection. Toutefois ce point mérite attention, car il touche déjà à la définition même de l'ordinaire et à son histoire, et risque d'entretenir un certain flou, ou même une ambiguïté, sur la nature liturgique de l'ordinaire et sur ses liens avec les ordines. Ainsi l'ordinaire, bien que n'étant pas, tout comme le coutumier, un livre liturgique au sens strict, doit-il être considéré comme un livre participant à la liturgie, puisqu'il codifie, réglemente les usages d'une communauté monastique, d'une cathédrale, d'un diocèse, entre autres. Dans un article récent (qui complète l'ouvrage de A.-G. Martimort), E. Foley ${ }^{4}$ parle, à juste titre me semble-t-il, de livre servant à la préparation du culte puisque, effectivement, l'ordinaire est à partir d'une certaine époque le complément indispensable aux livres liturgiques : il expose l'ordonnance de chaque office, de chaque célébration, établissant ainsi les liens entre les différents acteurs et leurs livres respectifs.

3. D. Nebbiat-Dalla Guarda, "Les listes médiévales de lectures monastiques", dans Revue bénédictine, t. 96, 1986, p. 271-326.

4. E. FoLEY, "The Libri ordinarii : An Introduction", dans Ephemerides liturgicae, t. 102,1988, p. $129-137$. 
Avant de passer à l'histoire même du livre et à l'affinement de sa typologie, voyons les trois grandes caractéristiques de la définition d'un ordinaire : tout d'abord, l'ordinaire suit de façon très précise le déroulement de l'année liturgique (le temporal et le sanctoral peuvent être mêlés ou distincts selon les cas), et c'est dans ce cadre temporel que sont décrites toutes les actions liturgiques propres à tel ou tel jour (notamment les processions) ; ensuite, l'ordinaire ne donne que l'incipit des pièces liturgiques dans l'ordre du déroulement des célébrations (cette caractéristique est à mettre en rapport avec sa fonction : il sert à celui qui doit diriger le déroulement des célébrations ; enfin, l'ordinaire décrit l'usage, essentiellement local, d'une cathédrale, d'un monastère, d'une collégiale, ou bien d'un diocèse, d'une famille monastique, d'un ordre canonial, monastique ou mendiant. Cette dernière caractéristique le distingue d'ailleurs nettement des ordines romani, qui entendent décrire des rites plus ou moins romains destinés à être adoptés partout, mais le rapproche des coutumiers qui proposent des usages communs dans le cadre d'une congrégation monastique, par exemple.

\section{Histoire de l'ordinaire et essai de typologie affinée}

Dans son ouvrage, Mgr Martimort a donné une riche synthèse qui doit être complétée et enrichie par l'article de E. Foley (cf. supra, note 4), mais aussi par deux travaux plus anciens : l'introduction de Mgr Hänggi à l'ordinaire de Rheinau du XII ${ }^{e}$ siècle ${ }^{5}$, et surtout le mémoire inédit de Jean Dufrasne sur les ordinaires manuscrits des églises séculières conservés à la Bibliothèque nationale de Paris ${ }^{6}$.

Grâce à son travail approfondi et minutieux sur les témoins manuscrits (ses notices sont les dignes héritières de celles de Leroquais pour d'autres livres), J. Dufrasne, dans son introduction, pose des questions essentielles pour l'histoire de ce type de livre, son rôle dans la liturgie et propose en même temps des éléments qui permettent d'affiner la typologie de ces documents.

Alors que les ordines sont concentrés sur des actions, l'ordinaire se focalise plutôt sur l'organisation liturgique d'un lieu, et cela tout au long de l'année. Au moment où il se sépare du coutumier et où il devient indépendant, essentiellement au XII siècle, l'ordinaire est un instrument de codification et de recherche d'unité de traditions liturgiques propres à une famille religieuse, aux nouveaux ordres, à un diocèse. Or, pour acquérir cette autorité, il a besoin d'être séparé du coutumier où il est "noyé " dans la masse des usages de la vie monastique ou canoniale qui y sont décrits. A côté de ce rôle joué dans la recherche d'une unité liturgique à l'intérieur d'une même famille religieuse ou d'un diocèse, l'apparition de l'ordinaire s'explique aussi par des raisons d'ordre pratique : il fait le tri, réorganise, classe les nombreuses pièces (chants, lectures, oraisons) qui sont apparues au fur et à mesure des siècles et sont venues enrichir le trésor de la prière de l’Église.

Plusieur̃s auteurs l'ont relevé, l'âge d'or des ordinaires monastiques est le XIII $^{\mathrm{e}}$ siècle, pendant lequel on assiste à une sorte d'individualisation très poussée des liturgies locales, ou propres à tel ou tel ordre religieux. Au cours de ce siècle, puis aux $X_{1}{ }^{e}$ et $x^{e} v^{e}$ siècles, les ordinaires sont le reflet de la mosä̈que des liturgies locales qui s'affirment alors dans tout l'Occident, ce qui explique leur importance comme source historique et liturgique d'un diocèse et, probablement, la forte proportion de publications, voire d'éditions, d'ordinaires locaux.

\footnotetext{
5. A. Hängri, Der Rheinauer Liber ordinarius, Fribourg, 1957 (Spicilegium Friburgense, $1)$.

6. J. Dufrasne, Les ordinaires manuscrits des églises séculières conservés à la Bibliothèque nationale de Paris, Mémoire dactylogr., Institut supérieur de Liturgie, Paris, 1959.
} 
Il faut aussi insister sur un autre aspect qui touche à l'apparition des ordinaires. En effet, la rédaction d'un ordinaire n'est pas fortuite : elle accompagne une réforme ou fixe une liturgie, une tradition liturgique, à une époque (essentiellement le XIII siècle) où les nombreux offices nouveaux (que l'on ajoutait auparavant dans le coutumier (?) ou dans les livres liturgiques, sur des feuilles annexes ou en marge) devaient être insérés dans un manuscrit. On procède alors localement, ou dans le cadre d'un ordre religieux, à une sorte de mise à jour des pratiques liturgiques destinée à lever les incertitudes, à favoriser le déroulement facile des cérémonies et à éviter les erreurs, les hésitations, bref tout ce qui contribue à l'affirmation d'une liturgie propre à un diocèse, à une cathédrale, à un monastère, à un ordre religieux. D'une manière générale, on peut dire que cette fixation par écrit des usages liturgiques marque aussi un mouvement de restauration de la liturgie, notamment dans le cadre diocésain.

Enfin, et surtout, l'ordinaire fait partie d'un ensemble de "nouveaux livres" liturgiques, les livres de seconde génération (après le sacramentaire, l'antiphonaire, les ordines pour la première génération), qui comprend le pontifical (celui issu de la réforme du Pontifical romano-germanique du $\mathrm{x}^{\mathrm{e}}$ siècle), le bréviaire, le missel. L'ordinaire ne s'explique et ne se comprend que dans le cadre élargi de cet ensemble qui marque un tournant dans l'ecclésiologie de la liturgie et ses livres. Or, comme le P. Gy l'a bien montré à propos des livres de la liturgie, l'ordinaire doit être considéré comme le pivot du passage du système carolingien au système tridentin; il apparaît, en effet, au sein des livres de seconde génération, comme celui à partir duquel s'organisent les autres livres, et il est donc au cœur d'une nouvelle ecclésiologie de la liturgie ${ }^{7}$.

Mgr Martimort a raison de souligner que les ordinaires se différencient d'après la nature de la communauté qu'ils régissent, et donc de distinguer les ordinaires des cathédrales - et des églises qui en dépendent - des ordinaires des monastères, ou bien encore des ordinaires destinés à l'ensemble d'une congrégation monastique ou canoniale. Mais, à l'intérieur de ces grandes catégories, on peut affiner la typologie grâce à un examen minutieux des manuscrits eux-mêmes et déboucher sur une caractérisation de chaque ordinaire à partir du cadre de la célébration à laquelle il correspondait. J. Dufrasne a ainsi remarqué que certains ordinaires fournissent de longues rubriques, extrêmement précises, qui permettent la reconstitution des offices ou de la messe tels qu'ils étaient célébrés dans telle ou telle église ; d'autres, au contraire, sont peu prolixes en indications rubricales (dans ce cas, les rubriques font simplement le lien entre les différentes pièces euchologiques) et ne nous donnent que peu de renseignements sur les célébrations, par exemple sur le détail des processions. Les deux cas de figure peuvent aussi bien apparaître dans le cadre d'une liturgie cathédrale, ou diocésaine, que dans celui d'une liturgie d'une famille monastique précise. On remarque à ce propos que plus on avance dans le temps, plus les ordinaires se gonflent, aux $\mathrm{XIV}^{\mathrm{e}}, \mathrm{xv}^{\mathrm{e}}$ et même $\mathrm{xvI}^{\mathrm{e}}$ siècles, de détails rubricaux d'une précision extraordinaire, indiquant jusqu'au poids des cierges que l'on transporte en procession. Les premiers ordinaires, essentiellement au $\mathrm{XIII}^{\mathrm{e}}$ siècle, sont assez sobres dans ce domaine et ne comprennent en moyenne que 60 à 80 feuillets, cela quel que soit le contexte liturgique de leur usage. Il n'est pas rare en revanche, à partir du $\operatorname{xIV}^{\mathrm{e}}$, mais surtout au $\mathrm{Xv}^{\mathrm{e}}$ siècle, de trouver des ordinaires comprenant 200 feuillets et plus, principalement en raison du gonflement des rubriques.

L'examen approfondi des manuscrits permet d'aller encore plus loin dans l'affinement de la typologie, reflet de la variété des célébrations. Le manuscrit

7. P.-M. Gy, "Typologie et ecclésiologie des livres liturgiques médiévaux", dans $L a$ liturgie dans l'histoire, Paris, 1990, p. 81. 
Paris, Bibl. nat., lat. 13874, réalisé à Corbie dans la première moitié du XIr ${ }^{e}$ siècle, contient d'abord le coutumier de Cluny (abrégé de l'Ordo cluniacensis de Bernard de Cluny) puis, dans une seconde partie (fol. 104-174), l'ordinaire du monastère de Corbie, l'ensemble présentant une grande unité paléographique et codicologique ; ceci est à mettre en rapport avec l'adoption à Corbie des coutumes clunisiennes, liée au souci de préserver la liturgie du lieu. Le ms Bibl. nat., lat. 1237 (Tours, plutôt du $x v^{e}$ que du $x v^{e}$ siècle?) juxtapose quant à lui, également avec une grande unité codicologique et paléographie, l'ordinaire de la cathédrale (fol. 1-51) et les statuts synodaux (fol. 5lv-83v); je reviendrai sur le sens de cette juxtaposition à partir de la redéfinition au XIIr ${ }^{e}$ siècle d'une liturgie diocésaine. Autre exemple, le ms Bibl. nat., lat. 1234. (Uzès, début du $\mathrm{xIv}^{\mathbf{e}}$ siècle) présente des mentions qui montrent que l'on a affaire à un ordinaire composé pour l'usage d'un diocèse, sans affectation précise, pas même celle de la cathédrale, mais au contraire en vue d'une diversité d'utilisation à l'intérieur du diocèse. S'agit-il d'un exemplaire de voyage (portatif?) destiné à l'évêque, que celui-ci utilisait lorsqu'il célébrait dans les paroisses : grâce à son ordinaire, il pouvait suivre les usages de sa cathédrale, en les adaptant à la paroisse qu'il visitait. La taille de ce manuscrit, assez petite pour un ordinaire $(19,5 \times 15 \mathrm{~cm})$, le nombre de feuillets (32), ainsi que l'aspect codicologique très peu soigné paraissent être des arguments en faveur de cette hypothèse. Sur l'aspect extérieur des manuscrits que j'ai pu consulter (manuscrits des XIII ${ }^{e}$ et $\mathrm{XIV}^{\mathrm{e}}$ siècles conservés à la Bibliothèque nationale, mais il faudrait faire une étude systématique et exhaustive), je dirai que, dans l'ensemble, l'ordinaire n'apparaît pas comme un livre luxueux : il ne fait pas l'objet d'un soin particulier, dans son aspect matériel, son écriture ou sa décoration. Ce fait renforce l'idée d'un livre essentiellement d'ordre pratique, utilitaire, que l'on consultait très régulièrement (les feuillets souvent abîmés et usés prouvent que ces livres ont manifestement été manipulés), contrairement aux grands livres liturgiques contenant les textes sacrés, somptueusement décorés et écrits, qui servaient dans l'acte même de la célébration. Dans le même ordre d'idées, il n'est pas rare que les textes sacrés et les rubriques soient transcrits avec une même encre brune ou noire, sans distinction visuelle, ne serait-ce que par l'utilisation d'une encre rouge pour souligner les rubriques (comme cela se faisait pour les ordines ou les pontificaux).

Quelques manuscrits font toutefois exception à la règle, mais il s'agit dans la plupart des cas de manuscrits issus de milieux royaux ou pontificaux. Ainsi, le ms Paris, Bibl. nat., lat. 1435 (50 fol., $17,5 \times 11,5 \mathrm{~cm}$, fin $\times \mathrm{xrv}^{e}=$ début $\mathrm{xv}^{e}$ siècle), ordinaire de la chapelle du roi de France (Sainte-Chapelle), est-il transcrit dans une écriture impeccable avec, en tête de chaque célébration, une initiale filigranée à l'exécution très soignée et des rubriques écrites à l'encre rouge visuellement distinctes des textes sacrés. Sa qualité matérielle se démarque nettement de celle de la plupart des exemplaires de cathédrales ou de grands monastères, car il s'agit d'un ordinaire destiné à régler la liturgie de la chapelle du roi de France. Dans un autre milieu, le ms Bibl. nat., lat. 4162 A (réalisé vers 1365 en Italie centrale pour le cardinal Albornoz) est un des rares ordinaires médiévaux à présenter un frontispice richement décoré indiquant qu'il contient le texte de l'ordinaire de la curie romaine du temps d'Innocent III (entre 1213 et 1216). Son écriture est soignée et les rubriques transcrites à l'encre rouge. Mais, à la différence de l'ordinaire de la Sainte-Chapelle, il fut manifestement beaucoup utilisé car il présente de nombreuses traces de manipulation. Tous ces exemples, que l'on pourrait multiplier à volonté, montrent bien l'intérêt qu'il y a à scruter de près les manuscrits dans le but d'affiner la typologie, permettant de dépasser les catégories de base et d'émettre des hypothèses sur les différentes formes de célébration dans tel ou tel contexte ecclésial et liturgique. Les ordinaires sont, en effet, les témoins privilégiés d'une liturgie vivante, aussi bien du point de vue matériel que spirituel. 


\section{L'histoire de l'Église et de la liturgie dans les ordinaires}

Dans un bref chapitre intitulé "Importance culturelle des ordinaires", Mgr Martimort a rappelé que ces livres de la liturgie étaient essentiels pour l'historien du culte chrétien - surtout au plan local (dans le cadre par exemple d'une cathédrale, ou d'une église monastique précise); pour le liturgiste, pour le théologien aussi - à cause notamment de l'étude de la forme prise par les célébrations des sacrements (enrichissement des pièces, des actes liturgiques, leur signification pour l'administration de tel ou tel sacrement) ; pour l'historien de la musique - qui complète grâce aux ordinaires ses connaissances sur l'évolution du répertoire propre à telle ou telle fête et sur l'histoire de la notation; enfin, pour l'historien de l'architecture et l'archéologue qui y trouvent des éléments essentiels pour la reconstitution de la chronologie d'un édifice.

La récente publication du premier ordinaire de Saint-Denis (Paris, Bibl. Mazarine, ms 526, première moitié du xIII ${ }^{e}$ siècle, 195 fol.), accompagnée d'un long commentaire par E. Foley, est un très bon exemple de ce qu'un ordinaire peut apporter à l'histoire de l'architecture et de son aménagement intérieur, à partir du cas particulier de Saint-Denis ${ }^{8}$. Écrit vers 1234 , le manuscrit reflète, presque un siècle après, l'œuvre architecturale et liturgique de Suger ( $\dagger 1151$ ) et témoigne des conditions du culte dans l'église gothico-carolingienne jusqu'à la fin du xıII siècle, après les remaniements architecturaux entrepris à partir de 1231 et terminés après 1281. Il serait d'ailleurs intéressant de comparer systématiquement les ordinaires avec ce que l'on sait, pour tel ou tel édifice, de l'histoire de son architecture, car il y a fort à croire que des remaniements architecturaux ou des réaménagements intérieurs, voire des reconstructions, ont suscité la rédaction d'un ordinaire. Dans l'ordinaire de Saint-Denis, on décrit l'espace où se déployait la liturgie : l'autel majeur, les chapelles du chevet, le chœur, les oratoires de la nef et de la crypte, les tombres royales, les bâtiments claustraux, les alentours du monastère y compris le cimetière, les parcours des processions. Dans ce contexte, il apparaît clairement que c'est la mémoire du saint martyr Denis qui est à l'honneur et que la présence des tombes royales n'a pas particulièrement influencé la liturgie abbatiale ${ }^{9}$. Le manuscrit de la Mazarine est également le témoin de la codification des usages liturgiques propres à l'abbaye de Saint-Denis - caractérisés principalement par leur origine bénédictine -, de sa conception pseudo-dionysienne et de son caractère fondamentalement franc, cela depuis l'époque carolingienne. Bref, le manuscrit 526 de la Mazarine est bel et bien la source la plus importante pour la reconstitution du culte à l'abbaye royale au XIII siècle.

De son côté, le spécialiste de la topographie des villes et des villages du Moyen Age trouve dans les ordinaires de précieux renseignements, notamment grâce aux descriptions des processions à travers les cités.

Pour les $\mathrm{xrv}^{e}$ et $\mathrm{xv}^{e}$ siècles, et même pour le $\mathrm{XvI}^{e}$ siècle, les ordinaires sont éventuellement une source permettant de cerner certains aspects de l'expression de la religiosité populaire et du drame sacré, le cas échéant à travers des pratiques superstitieuses relatées dans les textes. On y trouve aussi, surtout dans les manuscrits du $\mathrm{xv}^{\mathrm{e}}$ siècle présentant une grande inflation rubricale, des éléments de curiosité contenus dans certaines descriptions, telle l'énumération des boissons offertes au clergé lors des longues processions.

Mais, au-delà de toutes ces données, l'étude des ordinaires liturgiques permet aussi une meilleure compréhension de certains moments de l'histoire de l'Église et

8. E. Foley, The First Ordinary of the Royal Abbey of Saint-Denis in France, Fribourg, 1990 (Spicilegium Friburgense, 32).

9. Voir également E. FolEY, "Saint-Denis revisited : The Liturgical Evidence", dans Revue bénédictine, t. 100, 1990, p. 532-549. 
de la liturgie. Je ne prendrai que deux exemples significatifs : tout d'abord la nouvelle conception de l'Église à partir de la curie romaine, au début du $\mathrm{XIII}^{\mathrm{e}}$ siècle, et la mise en place d'une liturgie bien particulière, puis la restauration de l'Église séculière au XIII ${ }^{e}$ siècle et la redéfinition de sa liturgie.

1. On sait que, dans la première moitié $d u$ XIII $^{e}$ siècle, Innocent III et ses successeurs ont donné une nouvelle importance à la liturgie de la chapelle papale - devenue depuis la réforme grégorienne indépendante de celle du Latran concrétisée par la confection de nouveaux livres liturgiques avec, d'abord, l'ordinaire de la curie (entre 1213 et 1216), réglant à la fois l'office et la messe, puis le pontifical et le missel. Dans ce nouveau système, l'ordinaire tient une place de premier choix car il est le livre à partir duquel on réorganise les autres livres de la liturgie.

On sait aussi qu'au même moment se développe l'identification ecclésiologique entre l'ecclesia romana et la curia romana ${ }^{10}$. En fait, on en est venu progressivement à considérer la chapelle papale comme la réalisation liturgique la plus authentique de l'Église romaine. On peut se demander si l'élaboration de l'ordinaire de la curie (le témoin le plus ancien étant le manuscrit du xiv ${ }^{e}$ siècle, Paris, Bibl. nat., lat. 4162 A ${ }^{11}$, qui ne reflète plus l'état primitif de l'ordinaire entre 1213 et 1216) a été faite en vue d'une diffusion liturgique hors de la chapelle papale, dans le but de servir de modèle en d'autres lieux de l'Occident. Le titre du $\mathrm{XIV}^{\mathrm{e}}$ siècle porté par le ms lat. 4:162 A est : In nomine Domini. Incipit ordo Romanae Ecclesiae Curiae, quem consuevimus observare tempore Innocentii tertii papae et aliorum pontificum; ce titre était-il déjà dans l'ordinaire du temps d'Innocent III ? Suivre la liturgie de la chapelle papale en dehors du lieu même où elle était célébrée, telle était peut-être la raison initiale de la confection de ce livre et de la mise par écrit des usages liturgiques de cette chapelle. En tout cas, on remarque que le missel franciscain dérive d'un ordinaire pontifical de la première moitié du XIII ${ }^{e}$ siècle.

Il est intéressant de noter que certains éléments de l'ordinaire papal sont issus des ordines romani, revus par le Pontifical romano-germanique et les différentes refontes du pontifical romain. D'ailleurs, au xur ${ }^{\mathrm{e}}$ siècle, à Rome, la terminologie désignant le pontifical ou l'ordinaire est fluctuante et l'on confond souvent les deux livres. Le mot ordinarium avait encore à cette époque un sens assez indéterminé et on l'appliquait à des ouvrages hybrides qui n'étaient pas nécessairement des ordinaires. Ainsi, ordinarium pouvait aussi signifier ordo (Ordo romanus), et la richesse du vocabulaire employé, entre le $\mathrm{xIII}^{\mathrm{e}}$ et le $\mathrm{xv}^{\mathrm{e}}$ siècle, pour désigner un ordinaire : liber ordinarius (surtout dans les titres des manuscrits), ordinarium, ordinale, ordo, ordo ecclesiasticus, ordo officiorum, officium ecclesiasticum, breve, observantiae, consuetudines, liber caeremoniarum, directorium chori, montre qu'il se situe au confluent de bon nombre d'autres livres (ordines, pontificaux, bréviaires, coutumes, etc.).

2. II est bien connu que le xmI ${ }^{\mathrm{e}}$ siècle est celui de la restauration des structures de l'Église séculière, surtout en France, restauration qui se manifeste notamment par le renouveau de la liturgie dans le cadre diocésain. Ce renouveau liturgique n'a d'ailleurs été rendu possible qu'à partir du renforcement du pouvoir des évêques et des structures mêmes des Eglises locales : développement des chapitres cathédraux (élection de l'évêque), apparition dans les diocèses d'une véritable curie

10. Cf. P.-M. Gy, "La papauté et le droit liturgique aux $\mathrm{xII}^{\mathrm{e}}$ et $\mathrm{xIII}^{\mathrm{e}}$ siècles ", dans The Religious Roles of the Papacy : Ideals and Realities (1150-1300), Toronto, 1989 (Papers in Mediaeval Studies, 8), p. 229-245.

11. Sur cet important manuscrit pour l'histoire de la liturgie, cf. S.J.P. VAN DrJK, The Ordinal of the Papal Court from Innocent III to Boniface VIII and Related Documents, Fribourg, 1975 (Spicilegium Friburgense, 22). 
épiscopale, renforcement du pouvoir économique, meilleure emprise sur le cadre urbain (construction des grandes cathédrales, etc.).

Les synodes diocésains, déjà réactivés à la fin du XII $^{\mathrm{e}}$ siècle, suivis dans une partie des cas, au moins, de visites pastorales, constituent dans ce cadre un instrument de réforme et d'action entre les mains des évêques. D'année en année, ces réunions étaient l'occasion, entre autres, de l'élaboration et de la mise en place d'une véritable législation diocésaine permettant l'adaptation de règles générales aux situations concrètes, y compris dans le domaine de la liturgie. Or, on a pu constater un rapport fréquent entre les statuts synodaux du XIII $^{\mathrm{e}}$ siècle et la rédaction des ordinaires. Par exemple, à Angers en 1261, les statuts de Nicolas Gellent (1260-1281) précisent "Que l'on possède l'ordinaire et fasse l'office d'après lui. Nous ordonnons que chaque église soit en possession du livre appelé ordinaire que les prêtres consulteront chaque jour avant de commencer vêpres, afin de célébrer et d'accomplir ces mêmes vêpres, matines et l'office du jour suivant selon les indications de l'ordinaire " ${ }^{12}$. Mais, déjà dans la première moitié du XIII ${ }^{e}$ siècle à Paris, les statuts d'Eudes de Sully stipulent que l'ordinaire du prêtre de paroisse doit être conforme à celui de la cathédrale, première église du diocèse. On le voit, les prescriptions des statuts synodaux permettent souvent de préciser la date de confection des ordinaires. Dans plusieurs cas, comme dans celui de Mende, de Guillaume Durand, l'ordinaire distingue ce que l'on doit faire dans la cathédrale d'un côté et dans les paroisses du diocèse de l'autre. Ainsi que l'a montré le P. Gy ${ }^{13}$, l'ordinaire de Mende, et il n'est pas le seul dans ce cas, semble avoir été rédigé à la fois pour la cathédrale et pour l'ensemble du diocèse. Allant de pair avec les statuts synodaux, certains ordinaires, tel celui de Mende, ne décrivent pas seulement ce qu'on a coutume de faire (auquel cas ils ne seraient que la simple mise par écrit de la coutume de la cathédrale), ils marquent aussi une intervention d'autorité dans le choix des pièces et notamment des rites. Cela indique que certains ordinaires ont été soit corrigés, soit rédigés, à la même époque que les constitutions synodales dans l'intention de régler la liturgie du diocèse. On entend par là veiller à la bonne célébration de la liturgie, à la fois à la cathédrale et dans le diocèse, et résoudre certains problèmes posés par des pratiques locales. Bref, l'ordinaire "légifère " en matière de culte, comme les statuts synodaux le font dans d'autres domaines ; la juxtaposition, dans certains manuscrits, de l'ordinaire et des statuts synodaux prend alors tout son sens. La tenue de synodes diocésains peut donc être, selon les cas et les circonstances, l'occasion de la rédaction d'un ordinaire, ou bien de la révision d'un exemplaire déjà existant, voire de son aménagement dans le sens de l'élaboration d'une législation liturgique.

Éric Palazzo

12. La traduction est celle de Joseph AvrIL, Les statuts synodaux français du XIII siècle, t. III : Les statuts synodaux angevins de la seconde moitié du XIII siècle, Paris, 1988, p. 73. Toujours à Angers, un canon des statuts complémentaires de 1314 précise les modifications qu'il convient d'apporter à l'ordinaire des paroisses après l'institution dans le diocèse de l'octave de la Toussaint.

13. P.-M. Gx, "L'ordinaire de Mende. Une cuvre inédite de Guillaume Durand l'Ancien 》, dans Liturgie et musique, $I X^{e}-X I V^{e}$ siècles, Toulouse, 1982 (Cahiers de Fanjeaux, 17), p. 239-249. 